kenntnisreicher und kenntnisarmer Populationen zu vergleichen. Dafür sind auch experimentelle Ansätze nötig. Zur Einordnung des Niveaus des Faktenwissens in Deutschland sind außerdem international vergleichende Studien erforderlich.

Sämtliche Analysen von Befragten mit Migrationshintergrund kranken an der geringen Befragtenzahl; um hier Abhilfe zu schaffen, bedarf es stark überproportionaler Samples, da ansonsten multivariate Analysen mit vernünftiger Fallzahl kaum möglich sind. In Bezug auf Migranten wären zudem Panelerhebungen sinnvoll, um Kausalitätsprozessen zwischen Wissenserwerb, Identifikation mit dem Aufnahmeland und Erwerb der Staatsangehörigkeit des Aufnahmelandes auf die Spur zu kommen.

Insgesamt stellt sich die Ermittlung politischen Wissens in der Bevölkerung, der Ursachen und Folgen als zwar diffiziles, aber auch äußerst interessantes, wichtiges und im Hinblick auf das Erkenntnisinteresse lohnendes Forschungsgebiet dar, das in Deutschland noch einen erheblichen Ausbaubedarf hat.

\title{
Was bewegt Politiker mit Migrationshintergrund? Befunde aus deutschen Großstädten*
}

\author{
Constanze Schmitz und Andreas M. Wüst
}

„Türken, bringt euch mehr ein!“, forderte die CDU-Politikerin Aygül Özkan im April in einem Interview mit Spiegel Online. ${ }^{1}$ Wenige Tage zuvor war die Politikerin mit türkischem Migrationshintergrund eine Stufe auf der Karriereleiter nach oben geklettert. Christian Wulff, damals noch Ministerpräsident in Niedersachsen, bot der Abgeordneten der Hamburger Bürgerschaft den Posten der Sozialministerin in seinem Kabinett an. Damit wurde Özkan die erste türkischstämmige Ministerin Deutschlands - aus den Reihen der CDU. Das erregte Aufsehen, und obwohl sie Sozialministerin werden sollte, behandelten die vielen Fragen von Journalisten, denen sich Frau Özkan stellte, nicht das breite Spektrum des Politikfelds Soziales, sondern es ging fast ausschließlich um ihre Person und vermeintliche Gruppenzugehörigkeit. Wie kommt eine Muslimin dazu, in einer christlichen Partei politisch aktiv zu sein? Müsste sie nicht eigentlich bei der SPD oder bei den Grünen sein? Welche Integrationspolitik gedenke sie zu machen, und was hielt sie vom Integrationsstatus ihrer „Landsleute"?2

* Die Forschungsanstrengungen wurden durch die Volkswagen-Stiftung ermöglicht. Dies wird von den Autoren dankbar angezeigt.

1 Vgl. das Interview Aygül Özkans mit Anna Reimann, Neue Ministerin Özkan - „Türken, bringt euch mehr ein“, in: Spiegel Online vom 23. April 2010, http://www.spiegel.de/politik/deutschland/0,1518,690702,00.html (Abruf am 28. November 2011).

2 Vgl. ähnliche Inhalte bei Ulrich Exner, Hilfe, diese Muslima ist gar keine Christin!, in: Welt Online vom 26. April 2010, http://www.welt.de/politik/deutschland/article7346946/Hilfe-dieseMuslima-ist-gar-keine-Christin.html; „Es lohnt sich, sich anzustrengen“, in: Berliner Morgenpost vom 27. April 2010, http://www.morgenpost.de/printarchiv/seite3/article1296879/Es-lohntsich-anzustrengen.html; Stephanie Jungholt, Frau Özkan, warum sind Sie als Muslimin in der 
Das Spiegel-Interview verdeutlicht die mangelnde Selbstverständlichkeit, mit der Bürger mit Migrationshintergrund in die politische Elite Deutschlands aufrücken. Immer noch sind wenige Bürger mit ausländischen Wurzeln in einer deutschen Partei politisch aktiv oder machen politische Karriere, auch wenn sich ihre Anzahl seit den 1990er Jahren vervielfacht hat. ${ }^{3}$ Der Grundtenor der Reaktionen auf die Ernennung Aygül Özkans zur Ministerin ähnelt den Reaktionen auf den Grünen-Politiker Cem Özdemir, der 1994 die bundespolitische Bühne betrat und fortan als eine Art türkischer Botschafter im Parlament angesehen wurde. ${ }^{4}$ Der Vergleich mit Özdemir ist insofern instruktiv, als die Berufung in ein Staatsamt einen ähnlich weitreichenden Schritt in der Hierarchie politischer Funktionen darstellt. Hat man sich mittlerweile an so manchen Abgeordneten mit Migrationshintergrund gewöhnt, wird zumindest von Teilen der Öffentlichkeit eine Türkischstämmige und Muslimin, die für die größte christlich ausgerichtete Partei in Deutschland ein Staatsamt wahrnimmt, als zumindest gewöhnungsbedürftig angesehen. Die Reaktionen auf Sozialministerin Özkan weisen auf mögliche Barrieren hin: Persönliche Qualifikation, politische Einstellungen und Engagement könnten aus diesem Blickwinkel für die Wahrnehmung von Mandaten und Ämtern nicht genügen. Was also zeichnet diejenigen Personen mit Migrationshintergrund aus, die sich in Parlamenten engagieren (können)? Was sind die Gründe für ihr Interesse an politischer Arbeit, aus welchen Gründen entscheiden sie sich für die jeweiligen Parteien, wie erreichen sie ihre Ziele und welche Erfahrungen machen sie auf diesem Weg? Um diese Fragen geht es im vorliegenden Beitrag über Abgeordnete mit Migrationshintergrund in deutschen Stadtparlamenten.

\section{Politiker mit Migrationshintergrund als Gegenstand der Forschung}

Die wissenschaftliche Beschäftigung mit politischen Akteuren mit Migrationshintergrund hat in den vergangenen Jahren zugenommen. Immer stärker schenken Forscherteams dieser Personengruppe Aufmerksamkeit und beleuchten die politische Partizipation und Repräsentation in europäischen Einwanderungsgesellschaften. ${ }^{5}$ So konnten etliche Erkenntnisse über Wähler mit Migrationshintergrund ${ }^{6}$, die Form der Repräsentation, die Politiker mit Migrationshintergrund und auch die Inhalte ihrer Parlamentsarbeit gewonnen werden. Wir

CDU?, in: Bild.de vom 21. April 2010, http://www.bild.de/regional/hannover/interview/muslimin-cdu-12273906.bild.html (jeweils Abruf am 28. November 2011).

3 Vgl. Andreas M. Wüst / Dominic Heinz, Die politische Repräsentation von Migranten in Deutschland, in: Markus Linden / Winfried Thaa (Hrsg.), Die politische Repräsentation von Fremden und Armen, Baden-Baden 2009, S. $201-218$.

4 Vgl. Cem Özdemir, Ich bin Inländer. Ein anatolischer Schwabe im Bundestag, München 1997, S. $91 \mathrm{f}$.

5 Zum Forschungsstand und weiteren Verweisen Karen Bird / Thomas Saalfeld / Andreas M. Wüst (Hrsg.), The Political Representation of Immigrants and Minorities. Voters, Parties and Parliaments in Liberal Democracies, London 2011.

6 Vgl. Lise Togeby, Migrants at the Polls: An Analysis of Immigrant and Refugee Participation in Danish Local Elections, in: Journal of Ethnic and Migration Studies, 25. Jg. (1999), H. 4, S. 665 - 684; Johannes Bergh / Tor Bjorklund, The Political Representation of Immigrants in Oslo and Copenhagen: A Study of Electoral Systems and Voting Behaviour, in: Comparative Social Research, 22. Jg. (2003), S. $101-121$; Andreas M. Wüst, Wahlverhalten und politische Repräsentation von Migranten, in: Der Bürger im Staat, 56. Jg. (2006), H. 4, S. 228 - 234. 
wissen mehr über ihre parlamentarische Präsenz in Deutschland und über die Anteile in den politischen Lagern. ${ }^{7}$

Bei der Analyse der Fragetätigkeiten von Abgeordneten mit Migrationshintergrund zeigte sich $^{8}$, dass diese Parlamentarier ein breites Policy-Spektrum abdecken. Gemein ist den Themenbereichen jedoch, dass sie häufig auch migrationsspezifische Probleme behandeln. Diese Beobachtung wird durch die hohe Zahl der Migrationsbezüge in individuellen Anfragen von Parlamentariern mit Migrationshintergrund untermauert. Diese nehmen im Zeitverlauf sogar zu. ${ }^{9}$ Ebenso erhöht ein sichtbarer Migrationshintergrund den Anteil von Migrationsbezügen in Anfragen. Nehmen die Parlamentarier Positionsrollen ein, verlieren sich die migrationsspezifischen Besonderheiten individueller Parlamentstätigkeit. ${ }^{10}$

Abgeordnete sind allerdings nur eine Teilgruppe der politisch aktiven Bürger. So berichtet Nedim Ögelman ${ }^{11}$ auf der Basis von 104 Interviews mit türkischstämmigen „organization leaders and policymakers“ in Deutschland vom Einfluss migrantenspezifischer Netzwerke und der Herkunftskultur auf ihre politischen Schwerpunkte und Karriereinteressen. Norbert Cyrus ${ }^{12}$ beschäftigt sich mit dem Einstieg von Migranten in die Politik ihres Einwanderungslandes und welchen Themen sich diese zuwenden. Die Basis seiner Untersuchung bilden 22 Interviews mit politisch aktiven Einwanderern aus zwölf europäischen Ländern. ${ }^{13}$ Cyrus unterteilt sie in zwei Typen: Jene, die über ihre Aktivität in Migrantennetzwerken zu politischem Engagement kamen und solche, die sich zuerst im „mainstream“ des Einwanderungslandes engagierten und danach im Bereich Migration politisch oder gesellschaftlich tätig wurden. Gleich welchen Weg die Einwanderer beschritten, befassten sie sich in aller Regel mit Migrationsthemen. Wenn sie vorher in migrationsspezifischen Netzwerken aktiv waren, blieben diese Themen einerseits für sie wichtig. Andererseits komme es vor, dass sie sich gemäß den Erwartungen ihrer Partei darum kümmerten ${ }^{14}$, auch wenn dies für sie persönlich keinen hohen Stellenwert hatte.

In Deutschland sind es Parteien, die politische Eliten bilden und rekrutieren, ja geradezu ein Monopol $^{15}$ bei der Nominierung von Kandidaten besitzen. Innerhalb der Parteien gel-

7 Vgl. Andreas M. Wüst / Dominic Heinz, a.a.O. (Fn. 3).

8 Vgl. ebenda; Andreas M. Wüst / Constanze Schmitz, Zwischen migrationsspezifischer Prägung und politischer Opportunitätsstruktur: Abgeordnete mit Migrationshintergrund in deutschen Parlamenten seit 1987, in: Georg Weißeno (Hrsg.), Bürgerrolle heute. Migrationshintergrund und politisches Lernen, Bonn 2010, S. 127 - 144; Andreas M. Wüst / Thomas Saalfeld, Abgeordnete mit Migrationshintergrund in Großbritannien, Frankreich, Deutschland und Schweden: Opportunitäten und Politikschwerpunkte, in: PVS, Sonderheft 44 „Politik als Beruf“, Wiesbaden 2010, S. $312-333$.

9 Vgl. Andreas M. Wüst / Constanze Schmitz, a.a.O. (Fn. 8).

10 Vgl. Andreas M. Wiust / Thomas Saalfeld, a.a.O. (Fn. 8).

11 Vgl. Nedim Ogelman, Documenting and Explaining the Persistence of Homeland Politics Among Germany's Turks, in: International Migration Review, 37. Jg. (2003), H. 1, S. 163 - 193.

12 Vgl. Norbert Cyrus, Where Does It Start, Why Does It Change? Trajectory Patterns of Immigrants Active in Mainstream Political Parties, in: Dita Vogel (Hrsg.), Highly Active Immigrants. A Resource for European Civil Societies, Frankfurt am Main 2008, S. 129 - 144.

13 Österreich, Belgien, Dänemark, Finnland, Frankreich, Griechenland, Irland, Italien, Malta, Niederlande, Portugal, Schweden und Großbritannien.

14 Vgl. Norbert Cyrus, a.a.O. (Fn. 12), S. 144.

15 Vgl. Robert Lorenz / Matthias Micus, Politische Seiteneinsteiger - Exoten in Parteien, Parlamenten, Ministerien, in: dies. (Hrsg.), Seiteneinsteiger. Unkonventionelle Politiker-Karrieren in der Parteiendemokratie, Wiesbaden 2009, S. 11 - 30, S. 19. 
ten eigene Regeln, die es beim Antritt einer politischen Karriere zu beachten gilt. Elmar Wiesendahl $l^{16}$ erklärt, dass es weniger auf das ehrenamtliche Engagement ankomme, sondern vielmehr Eigeninitiative und Selbstrekrutierung von Bedeutung seien. Es komme zum „paradoxen Effekt der ,sozialen Schließung "17, indem nur jene eine politische Karriere beginnen, die Parteimitglieder sind, diese Rolle aktiv ausgestalten und sich bereit erklären, ehrenamtliche Parteifunktionen oder Wahlämter zu übernehmen. Die Schließung sei umso deutlicher, je weiter man auf der Karriereleiter nach oben wolle. Letztlich hieße das, die politische „Ochsentour“ absolvieren zu müssen, was gleichbedeutend mit einer zeitintensiven Parallelkarriere ist ${ }^{18}$ und somit für Beschäftigte im öffentlichen Dienst einfacher zu bewerkstelligen sei als für Arbeiter und Selbständige. Dies entspricht der Erkenntnis Jens Borcherts, dass die politische Karriere in den meisten westlichen Demokratien strikten Mustern folgt und somit politische Ämter nicht willkürlich angenommen würden, sondern eine klare Ämterhierarchie zugrunde liege. ${ }^{19}$

In einigen Fällen handelt es sich bei Migranten in der Politik auch um Seiteneinsteiger, die aufgrund ihres Erfolgs im Beruf oder durch berufliche und gesellschaftliche Kontakte mit Parteien vor Ort von Funktionsträgern der Parteien gezielt angeworben wurden. Nicht selten steckt dahinter das Interesse einer Partei, die eigene Kandidatenliste für die kommende Wahl zu diversifizieren und damit für verschiedene Wählergruppen attraktiver zu gestalten. Grundsätzlich ist es für Seiteneinsteiger leichter, einen Listenplatz zu erhalten als eine Direktkandidatur. ${ }^{20}$ Dies ist nach Robert Lorenz und Matthias Micus auf die strategischen Überlegungen und die Einflussmöglichkeiten von Parteieliten auf die Listenzusammensetzung zurückzuführen. Für eine Direktkandidatur hingegen sei die Ochsentour meist zwingend, was sich auch daran zeige, dass Parteifunktionen für die Karrieresicherheit eines Abgeordneten wichtiger seien als lokale Ämter. ${ }^{21}$ Die Ochsentour könne lediglich durch fördernde Mentoren, oftmals bereits verdiente Positionsinhaber, umgangen werden. ${ }^{22}$

Nicht nur für die Betrachtung des Karrierewegs ist der theoretische Ansatz der politischen Opportunitätsstruktur interessant. Ruud Koopmans und Paul Statham ${ }^{23}$ sehen in diesem Konzept ein geeignetes Analysemodell für die Migrationsforschung. Es handele sich um beständige, aber nicht zwangsläufig formelle und permanente Dimensionen der politischen Umgebung. ${ }^{24}$ Diese Strukturen schafften Anreize für Bürger, sich an kollektivem

16 Vgl. Elmar Wiesendahl, Rekrutierung von Eliten in der Parteiendemokratie. Wer sind die Besten und wie setzen sie sich durch?, in: Oskar W. Gabriel / Beate Neuss / Günther Rüther (Hrsg.), Eliten in Deutschland. Bedeutung, Macht, Verantwortung, Bonn 2006, S. 94 - 113, S. 106.

17 Ebenda, S. 107.

18 Vgl. ebenda; Jens Borchert / Lutz Golsch, Deutschland: Von der „Honoratiorenzunft“ zur politischen Klasse, in: Jens Borchert (Hrsg.), Politik als Beruf. Die politische Klasse in westlichen Demokratien, Opladen 1999, S. 114 - 140, S. 125.

19 Vgl. ders., Politik als Beruf: Die politische Klasse in westlichen Demokratien, in: ebenda, S. 7 39, S. 27.

20 Vgl. Robert Lorenz / Matthias Micus, a.a.O. (Fn. 15), S. 19.

21 Vgl. ebenda.

22 Vgl. ebenda, S. 21.

23 Vgl. Ruud Koopmans / Paul Statham, Migration and Ehnic Relations as a Field of Political Contention: An Opportunity Structure Approach, in: dies. (Hrsg.), Challenging Immigration and Ethnic Relations Politics, Oxford 2000, S. 13 - 56.

24 Vgl. Sidney Tarrow, Power in Movement. Social Movements, Collective Action and Politics, Cambridge 1994, S. 85. 
Handeln zu beteiligen und beeinflussten Erfolg oder Scheitern des Handelns. Hierbei sind für Koopmans' und Stathams Ansatz die Charakteristika eines politischen Systems relevant: Politische Kultur, Institutionen und die organisatorische Struktur eines Staates. ${ }^{25}$ Miki C. Kittilson und Katherine Tate ${ }^{26}$ unterstreichen mit ihrer Untersuchung über die Integration von ethnischen Minderheiten in Parteien in den USA und Großbritannien die Relevanz dieses theoretischen Modells.

Während Koopmans und Statham sich ausschließlich für die Strukturen auf der MakroEbene interessieren, berücksichtigen Kittilson und Tate neben der Makro-Ebene, dem Rechtssystem und dem ideologischen Klima in einem Staat auch die Meso-Ebene: die politischen Opportunitäten innerhalb von Parteien. Gerade in Deutschland, wo Parteien quasi eine Monopolstellung bei der Rekrutierung und Repräsentation politischer Eliten einnehmen, ist diese von großer Bedeutung. Der Parteienwettbewerb, die Auswahlkriterien innerhalb der Parteien und die Offenheit im Rekrutierungsprozess stellen eine besondere Herausforderung für Einwanderer dar.

Die Betrachtung der Makro- und Meso-Ebene reicht allerdings nicht aus, um ein umfassendes Bild über die (Karriere-)Wege von Migranten in der Politik zu erhalten. Karen Bird, Thomas Saalfeld und Andreas M. Wüst ${ }^{27}$ beschreiben politische Opportunitätsstrukturen als Mobilisierung durch kollektive Identität und Kapazität einer Minderheit und der in Bezug darauf gegebenen Reaktion des politischen Gefüges sowie des Parteiensystems. Neben der Makro- und Meso-Ebene benennen sie in ihrem Modell allerdings auch Faktoren auf der Mikro-Ebene. Diese wirken vor allem auf die Möglichkeiten der kollektiven Identität und Kapazität der Mobilisierung ein. Solche Faktoren sind unter anderem die politische Sozialisation, die Sichtbarkeit des Migrationshintergrunds, die Größe der ethnischen Gruppe, die Kommunikation innerhalb der Gruppe sowie die sozialen beziehungsweise ethnischen Netzwerke und Strukturen, die aufgebaut wurden.

Die zitierten Arbeiten geben jedoch wenig Aufschluss darüber, was Ursachen und Auslöser politischen Engagements von Bürgern mit Migrationshintergrund sind, und ob dieser Personenkreis mit spezifischen Herausforderungen im Verlauf der Karriere konfrontiert wird.

\section{Datenbasis: Leitfadeninterviews mit Stadtparlamentariern}

Basis der folgenden Auswertung sind 24 Leitfadeninterviews, die zwischen 2006 und 2009 mit deutschen Parlamentarierinnen und Parlamentariern mit Migrationshintergrund geführt wurden. Die Abgeordneten waren Mitglieder von Stadtparlamenten deutscher Großstädte sowie von Landesparlamenten der Stadtstaaten. ${ }^{28}$ Von den Befragten gehörten fünf der CDU an, zwei der FDP, neun der SPD, fünf den Grünen, zwei der Linkspartei und ein Befragter war Mitglied einer anderen lokalen Partei. Die persönlich geführten Einzelinter-

25 Vgl. Ruud Koopmans / Paul Statham, a.a.O. (Fn. 23), S. 36.

26 Vgl. Miki C. Kittilson / Katherine Tate, Political Parties, Minorities and Elected Office: Comparing Opportunities for Inclusion in the U.S. and Britain, Center for the Study of Democracy, University of California, Irvine, 3. Januar 2004, http://repositories.cdlib.org/csd/04-06/ (Abruf am 4. August 2008).

27 Vgl. Karen Bird / Thomas Saalfeld / Andreas M. Wüst, Ethnic Diversity, Political Participation and Representation. A Theoretical Framework, in: dies. (Hrsg.), a.a.O. (Fn. 5), S. 1 - 22, S. 13.

28 Zur Wahrung der Anonymität der Befragten werden diese Städte hier nicht aufgeführt. 
views dauerten zwischen 25 und 120 Minuten. ${ }^{29}$ In einigen Fällen wurden wegen Erkenntnissen, die nach der Durchführung aller Interviews gewonnen wurden, noch einmal Rückfragen an einzelne Politiker per E-Mail oder Telefon gestellt.

Qualitative Interviews sind in der biografischen Forschung und in ethnografischen Forschungsprojekten üblich. ${ }^{30}$ Die Leitfadeninterviews, die dem vorliegenden Beitrag zugrunde liegen, waren so konzipiert, dass sie die Erfahrungen der politischen Elite der in Deutschland lebenden Migranten nach Themenbereichen erfassen sollten. Daher ist die Form eines themengeleiteten Interviews, das jedoch Raum für Ausführungen und Abschweifungen lässt, sehr gut geeignet. Während es einerseits zur Überprüfung theoriegeleiteter Hypothesen genutzt werden kann, dient es andererseits auch dafür, neue Erkenntnisse zu Tage zu fördern und unerwartete Informationen in einer nachfolgenden Analyse zu systematisieren.

Das Leitfadeninterview umfasste acht Teile. Einleitend wurden Fragen zur Person gestellt, beginnend mit dem Elternhaus und Migrationserfahrungen über Bildung und Ausbildung bis zur Bedeutung der Religion. Danach wurden die Abgeordneten zu ihrem politischen Engagement und dem Weg in die Politik befragt. Im dritten Abschnitt ging es um die Entscheidung für die Partei und die Erlebnisse innerhalb der Parteiorganisation. So wurden unter anderem Fragen zu Kandidaturen innerhalb der Partei und zu etwaigen Parteiämtern gestellt. Der vierte Block behandelte Kandidaturen für Mandate, wie es zur Mandatsübernahme kam und wie sich die Zusammenarbeit in der Fraktion und im Parlament gestaltet. Dem schlossen sich Fragen zur politischen Schwerpunktsetzung an. Der sechste Teil befasste sich mit dem Repräsentationsfokus und Repräsentationsstil: Die Abgeordneten sollten unter anderem Auskunft darüber geben, wen sie im Parlament vertreten wollen und welche Rolle einzelne Bürger und gesellschaftliche Gruppen (unter anderem Migrantengruppen) im Repräsentationsprozess spielen. Danach wurden die Befragten aufgefordert, eine Bewertung und einen Ausblick bezüglich ihrer politischen Arbeit zu geben, welche inhaltlichen Ziele sie noch erreichen wollen oder welche Ämter und Mandate sie in Zukunft anstreben. Abschließend konnten die Interviewpartner Anregungen geben oder Sichtweisen auf das Forschungsthema anmerken.

Mit 24 Interviews wurde etwa ein Fünftel der rund 140 Abgeordneten (Stand: Februar 2008) mit Migrationshintergrund in den 25 größten Städten Deutschlands ${ }^{31}$ befragt. Trotz dieses beachtlichen Anteils können die hier dokumentierten Ergebnisse angesichts der niedrigen Fallzahl keinen Anspruch auf Repräsentativität erheben. Die Autoren sind jedoch der Ansicht, dass sie ausreichen, um einerseits theoriegeleitete Annahmen zu plausibilisieren und um andererseits zu weiterer Hypothesenbildung mit Blick auf potenziell relevante individuelle oder institutionelle Faktoren für die Migrantenrepräsentation beizutragen.

29 In einem Fall wurden zwei Parlamentarier derselben Partei einer Stadt gemeinsam interviewt.

30 Vgl. Christel Hopf, Qualitative Interviews - ein Überblick, in: Uwe Flick / Ernst von Kardorff I Ines Steinke (Hrsg.), Qualitative Forschung. Ein Handbuch, Hamburg 2008, S. 349 - 360, S. 349.

31 Parlamentarier mit Migrationshintergrund in den 25 größten Städten, einschließlich der Berliner Bezirksparlamente, sowie der Landesparlamente von Berlin, Hamburg und Bremen. Eine aktualisierte Datenbasis, die unter Leitung von Karen Schönwälder (MPI-MMG in Göttingen) für sämtliche Großstädte erstellt wurde, geht von 190 Abgeordneten mit Migrationshintergrund aus. Vgl. Matthias Kamann, In der deutschen Politik fehlen Migranten, in: Welt Online vom 28. Juli 2011, http://www.welt.de/print/welt_kompakt/print_politik/article13511672/in-der-deutschenpolitik-fehlen-migranten.html (Abruf am 28. November 2011). 
Durch die Auswertung einzelner Aussagen und Erfahrungsberichte eröffnen sich neue Perspektiven für die Bearbeitung des Forschungsthemas, und es können möglicherweise auch neue Hypothesen generiert werden.

\section{Migrationserfahrungen, Sozialisation und Opportunitäten}

Um das politische Handeln und den Karriereverlauf von Parlamentariern mit Migrationshintergrund besser verstehen zu können, wurden im Rahmen der Interviews insbesondere biografische Aspekte und die Aufnahme der Politiker in Partei und Fraktion beleuchtet. Während der Auswertung dieser Gespräche kristallisierten sich drei Themenkomplexe als besonders interessant für ein besseres Verständnis der Motivation und Arbeit von Abgeordneten mit Migrationshintergrund heraus: Migrationserfahrungen, politische Sozialisation und politische Opportunitäten.

Mit der Schilderung der Migrationserfahrungen wird die individuelle Ausgangslage der Abgeordneten erfasst, die oftmals Einfluss auf die politische Sozialisation der Befragten hat. Zwanzig Parlamentarier gehörten der ersten ${ }^{32}$, vier der zweiten Migrantengeneration an und wurden zu Beginn der Interviews aufgefordert, über ihre spezifischen Erfahrungen bei der Aus- und Einwanderung zu berichten. Bei Einwanderern der ersten Generation waren dies in der Regel eigene, zumeist prägende Erfahrungen. Diese Berichte wurden, insbesondere bei Migranten der zweiten Generation, durch familiäre Erfahrungen, also der Geschichte der Eltern oder naher Verwandter, ergänzt. In der Forschung wird mit Bezug auf die Migrationsentscheidung und -ursache zwischen eher freiwilliger Einwanderung - zum Beispiel zur Bildung und Ausbildung oder Erwerbsarbeit - und eher erzwungener - vor allem aus politischen, teils aber auch aus religiösen oder kulturellen Gründen - unterschieden. ${ }^{33}$ Dies legt nahe, dass bei unfreiwilliger Auswanderung die Erfahrung von Gewalt ein Auslöser für das politische Engagement sein könnte.

Politische Sozialisation kann in verschiedenen Kontexten und auf verschiedene Weise erfolgen. Einige Abgeordnete berichteten über starkes politisches Interesse im Elternhaus. Aktivitäten im eher vorpolitischen Raum gehören ebenfalls zur Sozialisation: Nicht selten sind die Parlamentarier Gewerkschaftsmitglieder oder engagier(t)en sich in Vereinen oder Verbänden. Dieses Engagement führt oft zu Kontakten mit verschiedenen aus- und inländischen Organisationen und hat offenbar auch Einfluss auf die grundsätzlichere politische Ausrichtung. Eine mögliche Folge des soziopolitischen Engagements ist die gezielte Anwerbung durch Parteien. Die politische Sozialisation eröffnet somit Opportunitäten in der Politik. Allerdings muss Engagement nicht zur Anwerbung führen, und nicht jedes Angebot muss angenommen werden. Wie die Interviews zeigen, ist es häufig der eigene Wunsch, parteipolitisch oder parlamentarisch aktiv zu werden. Insofern gibt es Muster der Eigenwie der Fremdmotivation.

Parteien sind einerseits durch Wahlsysteme institutionellen Rahmenbedingungen unterworfen, andererseits setzen sie sich durch formale und informale Nominierungsverfahren

32 Acht von diesen sind bereits als Kinder nach Deutschland gekommen und hier aufgewachsen. Sie könnte man auch zur „Generation 1,5“ zählen.

33 Vgl. Annette Treibel, Migration in modernen Gesellschaften. Soziale Folgen von Einwanderung, Gastarbeit und Flucht, Weinheim 1999, S. 20. 
eigene Strukturen. ${ }^{34}$ Diese Rahmenbedingungen haben entscheidenden Einfluss auf die strategischen Überlegungen der Parteien wie auch der politisch aktiven Migranten. Es wurde bereits erwähnt, dass das theoretische Modell der politischen Opportunitätsstrukturen geeignet ist, Gelegenheiten und Barrieren für Einwanderer in Parteien und politischen Systemen zu identifizieren. ${ }^{35}$ Auch in den hier ausgewerteten Interviews wurde nach der Opportunitätsstruktur gefragt. Die Befragten berichteten über ihre Erfahrungen innerhalb der Partei und im politischen System. Die Abläufe, Erfolge und Misserfolge bei Kandidaturen für Parteiämter und Mandate lassen Rückschlüsse auf die institutionellen und gesellschaftlichen Hürden zu, mit denen politisch aktive Migranten in Deutschland konfrontiert sind.

\section{Auswertung der Interviews: Erfolgsmuster und Hindernisse}

Bei der Auswertung der Interviews konnten Erfolgsmuster und Hindernisse auf individueller Ebene festgestellt werden. Migranten machten in den verschiedenen Parteien teils ähnliche Erfahrungen, was Offenheit, Nominierung für Wahllisten oder Ämtervergabe betrifft. Zusätzlich zur Betrachtung und Gegenüberstellung der individuellen Aussagen erschien vor allem aufgrund der Fallzahlen eine Differenzierung der Interviewten in zwei politische Lager sinnvoll: SPD, Grüne und Linkspartei (linksorientierte Parteien) sowie CDU und FDP (bürgerlich-konservative Parteien). Von den 24 Befragten gehörten sieben dem bürgerlichkonservativen und 17 dem linksorientierten Lager an.

\subsection{Migrationserfahrungen}

Bereits bezüglich der Migrationserfahrungen ergaben sich Unterschiede nach Parteilager. So berichtete keiner der bürgerlich-konservativen Abgeordneten über das Erleben von Gewalt im Aus- oder Einwanderungsland. Unter den linksorientierten Befragten waren es hingegen sechs Parlamentarier, die von eigenen oder familiären Gewalterlebnissen sprachen. Dieser höhere Anteil kann daher rühren, dass insbesondere in der Türkei, aber auch in Griechenland, im linken politischen Lager engagierte Bürger staatliche Repressionen erfuhren. Allen voran Türkischstämmige berichteten davon, sei es aufgrund der politischen Orientierung oder weil sie bereits im Herkunftsland Teil einer religiösen oder ethnischen Minderheit waren (Aleviten und Kurden). Jene drei Parlamentarier, die aus Gründen des politischen Asyls nach Deutschland einreisten, sind entsprechend ebenfalls bei den linksorientierten Parteien zu finden.

\subsection{Politische und religiöse Sozialisation}

Hinsichtlich der Sozialisation war anzunehmen, dass politische Diskussionen oder ein politisches Engagement der Eltern Einfluss auf die Kinder hatte. In beiden Lagern bestätigte

34 Vgl. Lise Togeby, The Political Representation of Ethnic Minorities: Denmark as a Deviant Case, in: Party Politics, 14. Jg. (2008), H. 3, S. $325-343$.

35 Vgl. Miki C. Kittilson / Katherine Tate, a.a.O. (Fn. 26); Ruud Koopmans / Paul Statham, a.a.O. (Fn. 23); Karen Bird/ Thomas Saalfeld / Andreas M. Wüst, a.a.O. (Fn. 27). 
sich ein Zusammenhang zwischen dem politischen Interesse der Eltern und dem Engagement der Kinder. Einige Politiker berichteten, dass sich vornehmlich die Väter mit der Politik des Herkunftslandes auseinandersetzten, die anschließend zuhause diskutiert wurde. Dagegen spielten die politischen Ereignisse im Einwanderungsland eine geringere Rolle. Jeweils mehr als die Hälfte der Befragten gab an, dass die Eltern politische Ereignisse verfolgten, doch selbst politisch aktiv waren nur wenige.

Deutliche Unterschiede zwischen den bürgerlich-konservativen und den linksorientierten Befragten zeigten sich bezüglich der Rolle der Religion. Über die Hälfte im bürgerlichkonservativen Lager maß ihr nicht nur eine persönliche, sondern eine persönliche und politische Bedeutung bei. Lediglich ein FDP-Politiker gab an, dass die Religion gar keine Rolle spiele. Bei den Linksorientierten sagten hingegen nur zwei Abgeordnete, dass die Religion für sie persönlich und politisch wichtig sei. Mehrheitlich lautete die Antwort, dass die Religion ein Teil der Kultur sei oder aber keine Rolle spiele. Gerade im linken Lager gehörten mehr Befragte der alevitischen Glaubensgemeinschaft an. Sie beschrieben sich als weltoffener, nicht streng religiös, und der humanistischen Bildung sei im Elternhaus ein hoher Stellenwert zugeschrieben worden.

Betrachtet man das vorpolitische Engagement, zeigt sich, dass auch bei Migranten von einem Zusammenhang zwischen gesellschaftlicher und politischer Partizipation ausgegangen werden kann. ${ }^{36}$ Nur ein Befragter gab an, vor dem Eintritt in die Politik nicht gesellschaftlich oder politisch aktiv gewesen zu sein. Insgesamt war das Engagement der Befragten in ethnischen Vereinen gering. Vielmehr handelte es sich um Mitgliedschaften in Gewerkschaften, sozialen und kirchlichen Organisationen oder Studentenvertretungen. Während im bürgerlichen Lager Aktivitäten in sozialen und religiösen Organisationen überwogen, kamen Angehörige des linken Lagers über die Gewerkschaft zur Politik oder waren bereits im Auswanderungsland Mitglied einer Partei beziehungsweise politisch aktiv gewesen.

\subsection{Parteieintritt und Aufnahme}

In den Interviews gaben die meisten Befragten zu Protokoll, eigenmotiviert in eine Partei eingetreten zu sein. Andere wurden gezielt als Kandidaten angeworben und kamen auf diesem Weg zur Partei. Während im linken Lager die Zahl derer, die von sich aus auf eine Partei zugegangen waren, überwiegt (elf von sechzehn Befragten), kamen bei den konservativen Politikern mehr (vier von sieben Befragten) durch gezielte Anwerbung zur Partei.

Einzelaussagen geben Hinweise auf die Hindernisse, die den Parteieintritt oder womöglich auch das allgemeine gesellschaftliche Engagement erschwert haben. Es werden vor allem kulturelle Unterschiede und Kommunikationsprobleme genannt. Zwei Befragte türkischer Abstammung thematisierten in diesem Zusammenhang die vermeintliche Zurückhaltung vieler Türkischstämmiger. Sie warteten darauf, angesprochen und eingeladen zu werden, während auf der anderen Seite die Haltung der Deutschen oftmals genauso

36 Vgl. Jan W. van Deth, Soziale und poltische Beteiligung: Alternativen, Ergänzungen oder Zwillinge?, in: Achim Koch / Martina Wasmer / Peter Schmitt (Hrsg.), Politische Partizipation in der Bundesrepublik Deutschland. Empirische Befunde und theoretische Erklärungen, Opladen 2010, S. $195-219$. 
passiv sei: Jeder Migrant dürfe mitmachen, „sie sollten einfach kommen, wenn sie wollten“, gab ein Befragter die Haltung der Parteien wieder. Ein Stadtrat türkischer Herkunft wies darauf hin, dass der zwischenmenschliche Umgang in Deutschland anders sei. Die Parteitreffen hätten hier nicht den familiären Charakter wie in der Türkei. Dort stünde der private Austausch stärker im Vordergrund; gehöre man zur Partei, gehöre man zu einer Art Familie. Demgegenüber sei der Umgang der deutschen Parteikollegen untereinander kühl; aus einer türkischen Perspektive heraus erscheine dies als Desinteresse. Nur selten gehe man miteinander aus oder rede über Privates.

Während der Interviews entstand der Eindruck, dass Seiteneinsteiger weniger gut in die Parteiorganisation, insbesondere in Form von Parteiämtern, eingebunden sind. Bei der Auswertung bestätigte sich dies jedoch nur für die linksorientierten Parteien. Keiner der fünf rekrutierten Befragten hatte ein Parteiamt inne. Von den übrigen zwölf bekleideten acht ein Parteiamt. Nach Auskunft der Befragten hat dies vor allem zeitliche Gründe oder beruht auf Desinteresse an einer Profilierung in der Parteiorganisation. Lediglich ein befragter Seiteneinsteiger des konservativen Lagers berichtete frustriert über seine unerfüllten parteilichen Ambitionen: Er habe anfangs zwar Interesse an Parteiämtern gezeigt, sei aber aufgrund innerparteilicher Widerstände für die gewünschten Positionen nicht nominiert worden.

Auf Basis der Auskünfte der insgesamt geringen Zahl interviewter Politiker mit Migrationshintergrund sollte nicht geschlussfolgert werden, dass tatsächlich der Hintergrund das Haupthindernis für den Erwerb eines Parteiamts ist. Einige Befragte berichteten, dass die Ethnie sehr wohl immer eine Rolle spiele und sie dies in „spitzen“ Kommentaren gelegentlich auch zu hören bekämen. Einzelne Berichte über „ethnisches Mobbing“ dürfen aber nicht dazu führen, den Hintergrund als zentrales Hindernis für die Karriere zu fixieren: Es kann der Migrationshintergrund sein, aber beispielsweise auch mangelnde Qualifikation, ideologische Distanz oder persönliche Antipathie, wahrscheinlich jedoch ein Zusammenspiel verschiedener Faktoren.

Unter den Migranten gibt es Seiteneinsteiger, die durch die Förderung von Mentoren schnell(er) zu einem Mandat oder Parteiamt kamen. So berichteten vier Befragte, darunter drei Frauen, dass ihnen die Hilfe und Rückenstärkung durch bekannte Politikerinnen und Politiker der Partei bei Nominierungen, aber auch beim persönlichen Durchhalten geholfen habe. Andere beklagten sich über mangelnde Protektion und brachten dies mit einem Gefühl des Ausgeschlossen-Seins in einen ursächlichen Zusammenhang.

Wenn die Offenheit der Partei gegenüber dem Engagement von Migranten angesprochen wurde, antworteten die Befragten im linken Lager mehrheitlich, dass es ihrer persönlichen Erfahrung nach keine Barrieren gäbe (elf von siebzehn). Lediglich ein Politiker berichtete über kulturelle Hindernisse, die übrigen fünf sahen die Probleme in ideologischen oder persönlichen Differenzen mit den Parteikollegen. Im konservativen Lager bestätigten vier der sieben Befragten das Vorhandensein von Barrieren, die sie auf persönliche oder kulturelle Differenzen zurückführten. Interessant sind in diesem Zusammenhang die ideologischen Hürden, die lediglich von den Befragten der linksorientierten Parteien angeführt wurden. Insbesondere von Abgeordneten der Grünen wird über einen hohen Anpassungsdruck an „ideologische Vorgaben“ der Partei berichtet.

Netzwerke der Migranten innerhalb von Parteien oder zwischen Fraktionen sind in den verschiedenen Städten unterschiedlich stark ausgeprägt. Persönliche Sympathien wirken gelegentlich als Motor für die interfraktionelle Zusammenarbeit. So gibt es in einem der 
Stadtparlamente eine Art Stammtisch der Abgeordneten mit Migrationshintergrund, von dem lediglich die (neu hinzugekommenen) Abgeordneten der Linkspartei ausgeschlossen zu sein scheinen. Andere sprechen von persönlichen Kontakten, die gemeinsame Initiativen erleichterten oder die Chance erhöhten, Unterstützung einer anderen Fraktion zu erhalten. Dies wirke aber auch umgekehrt: Persönliche Antipathie oder Distanz zwischen Fraktionen verhinderten eine manchmal sinnvolle Zusammenarbeit, wie es in einer anderen Stadt über die Fraktionen der Grünen und der SPD berichtet wurde. Alles in allem entstand im Rahmen der Befragungen nicht der Eindruck, dass migrationsspezifische, parteiübergreifende Netzwerke eine besondere Bedeutung in der parlamentarischen Praxis hätten.

\subsection{Kandidaturen um Mandate}

Vor allem bei der Vergabe von Listenplätzen scheint in beiden politischen Lagern „die Ethnie vor Qualifikation und Leistung“ zu zählen, wie es eine Abgeordnete ausdrückte. Und mehrere Befragte haben den Eindruck, dass insbesondere innerhalb der zweiten Generation eher ein Wettstreit um die Nominierung anstatt eine Kooperation der Kandidaten mit Migrationshintergrund bestünde. Mittlerweile engagieren sich mehr Einwanderer in den Parteien, und diese erhielten nicht mehr allein aufgrund ihres Migrantenstatus' einen Listenplatz. Die Antworten der Befragten zeigen, dass keine Parteiführung bei der Listenerstellung freiwillig mehr als einen aussichtsreichen Platz an einen Kandidaten mit Migrationshintergrund vergibt. In einem Fall berichtete ein Befragter über die Vergabe eines „inoffiziellen Quotenplatzes“ für Migranten. Auch er sah bei der Listenaufstellung eher den Proporz von Ethnie und Geschlecht als die Leistung berücksichtigt. Er setzte sich mit einer Kampfkandidatur zur Wehr und konnte sich - seiner Ansicht nach aufgrund der persönlichen Leistungsbilanz - als zusätzlicher Kandidat mit Migrationshintergrund auf einem aussichtsreichen Listeplatz behaupten.

\subsection{Auswahl der politischen Themenschwerpunkte}

Die inhaltlichen Schwerpunkte setzen die meisten der befragten Abgeordneten mit Migrationshintergrund in der Sozialpolitik: Gesellschafts-, Integrations-, Bildungs- und Familienpolitik werden am häufigsten behandelt. Ebenso sind viele Abgeordnete mit Migrationshintergrund in Ausschüssen vertreten, die sich mit inneren Angelegenheiten, also Verwaltung, Personal und Finanzen befassen. Zwar erstrecken sich die Schwerpunkte über alle Politikfelder, jedoch in keinem anderen sind die Migranten so stark vertreten wie in der Sozialpolitik. Insbesondere für Abgeordnete der FDP ist dies eine interessante Konstellation: Die beiden Befragten gaben an, Sozialpolitik und die Interessen von Einwanderern gerade in dieser Partei behandeln und voranbringen zu wollen. Sie kümmerten sich daher weniger um die traditionellen und personell stärker umkämpften Politikfelder der Liberalen, sondern widmeten sich besonders der Migrations- und Sozialpolitik. Hier werden offensichtlich Nischen besetzt, die einerseits dazu führen können, das programmatische Profil einer Partei zu erweitern; andererseits laufen Politiker Gefahr, Fremdkörper außerhalb des Mainstreams der Partei zu bleiben beziehungsweise zu werden. 
Unzufriedenheit mit der Mitgliedschaft in Parlamentsausschüssen wurde selten geäußert. Die meisten gaben an, dass ihre Wünsche berücksichtigt wurden. Nur wenige beklagten durchweg allerdings mit der Plausibilisierung, dass sie sich wie andere Neuparlamentarier „hinten anstellen“ müssten -, in einen nicht präferierten Ausschuss gekommen zu sein. Obwohl sich alle befragten Abgeordneten mit Migrationshintergrund mit den Politikbereichen Integration und Migration beschäftigten, taten es nicht alle in Form einer entsprechenden Ausschussmitgliedschaft. Während es für einige Befragte aufgrund ihres eigenen Migrationshintergrundes ganz selbstverständlich zu sein scheint, ihren Schwerpunkt auf Integrationsfragen zu setzen, sehen andere darin eher ein Zugeständnis auf Druck der eigenen Fraktion. So berichtete eine Befragte, in ihrer Fraktion sei es für Migranten nicht erwünscht, ein anderes Thema zu besetzen. Und ein Befragter, der in Deutschland geboren und aufgewachsen ist, keinen sichtbaren Migrationshintergrund und einen deutschen Elternteil hatte, wunderte sich, dass ihm die Fraktion ungefragt die Migrationspolitik „übertrug“. Manchmal entstehen beim Ersteinzug eines Einwanderers in ein Parlament aber auch migrationsspezifische Probleme ganz eigener Art: In Hessen kam es so zu Beginn der extrem kurzen 17. Wahlperiode des Landtags bei der SPD zur Ernennung zweier Fraktionssprecher für Migration.

\section{Migranten als Parlamentarier: Bestätigte Annahmen, neue Erkenntnisse}

Die Analyse der Interviews mit Migranten in Stadtparlamenten bestätigte etliche vermutete Zusammenhänge, erbrachte aber auch neue Ergebnisse, die für das Verstehen und die Erforschung ihres politischen Engagements nützlich sein könnten. So stellte sich heraus, dass sich Politiker verschiedener politischer Lager auch hinsichtlich bisheriger Lebenserfahrungen unterscheiden. Befragte mit Migrationshintergrund im linken Lager waren häufiger bereits im Auswanderungsland politisch engagiert und haben teilweise auch selbst politische Repressionen erfahren. Im Einwanderungsland sind sie folglich eher an Parteien interessiert, in denen Minderheitenschutz einen hohen Stellenwert einnimmt. Sie suchen meist selbst den Kontakt zu Parteien. In den bürgerlich-konservativen Parteien kommt es dagegen häufiger zu Rekrutierungen. Dies scheint vor allem aus strategischem Interesse zu geschehen, um die Liste durch Kandidaten mit Migrationshintergrund „aufzuwerten“. Den Kandidaten sind diese parteipolitischen Beweggründe durchaus bewusst. Aber sie nehmen die Möglichkeiten der Ausgestaltung und Instrumentalisierung einer solchen Funktionsrolle auch selbst wahr. So sei es dadurch unter anderem leichter, einen sicheren Listenplatz für sich zu reklamieren.

Die Auswertung bestätigt ferner, dass sich Politiker mit Migrationshintergrund dem Politikfeld Migration nicht vollends entziehen können und dies zumeist auch nicht wollen. Sie fungieren als Brückenbauer zwischen den Kulturkreisen, unabhängig davon, ob sie starke Verbindungen zu ihren ethnischen Gruppen pflegen oder nicht. Problematisch wird es jedoch, wenn die Aktivitäten der Abgeordneten mit Migrationshintergrund ausschließlich auf dieses politische Thema beschränkt bleiben. Eine solche Gefahr besteht vor allem bei den angeworbenen Seiteneinsteigern, die quasi als „Berufsmigranten“ gezielt für Wählergruppen mit Migrationshintergrund zuständig sein sollen.

Die häufige (gewollte oder auch ungewollte) Übernahme des Politikfelds Migration bedeutet für die Abgeordneten mit Migrationshintergrund in der Regel eine Mehrbelas- 
tung. ${ }^{37}$ Wenn sie andere politische Schwerpunkte setzen wollen, müssen sie zusätzlich Zeit investieren. Aufgrund der wachsenden Konkurrenz durch Kandidaten mit Migrationshintergrund ist es aber wichtig, sich auch in anderen Bereichen zu profilieren. Allerdings ist das Politikfeld Migration mittlerweile stark umkämpft. Die Aussagen über eine Ellbogenmentalität unter den Kandidaten mit Migrationshintergrund oder aber, dass ,allein Migrant zu sein" nicht mehr ausreiche, um aufgestellt zu werden, unterstreichen diese Entwicklung.

Innerhalb der Parteien existieren längst inoffizielle Quotenregelungen für die Vergabe von Listenplätzen. Migranten auf der Wahlliste zu haben, ist inzwischen für alle Parteien wichtig. Bislang ist zu beobachten, dass etliche Kandidaten mit Migrationshintergrund allein aufgrund ihrer Herkunft Vorteile bei der Listenplatzvergabe haben. Kandidaten kommen normalerweise selten ohne Absolvierung der Ochsentour auf einen vorderen Listenplatz. Aber mehr als ein Migrant oder, besser noch, eine Migrantin (die auch noch den Frauenproporz erhöht) auf den aussichtsreichen Plätzen soll es häufig auch nicht sein. Je mehr Einwanderer sich in Parteien engagieren, umso wichtiger könnte es für sie - und vor allem für die Seiteneinsteiger unter ihnen - werden, auch in der Partei verwurzelt zu sein und in der Parteiorganisation Ämter einzunehmen. Engagement auf den unterschiedlichen Ebenen der Partei sollte auch für Migranten von Vorteil sein, um nicht nur Mandate erlangen zu können, sondern auch bessere Voraussetzungen zu besitzen, um für politische Ämter nominiert zu werden.

Mitunter überraschend waren Aussagen etlicher Abgeordneter der Grünen, die über einen hohen Anpassungsdruck an die ideologische Linie der Partei berichteten. Allerdings wurde die Herkunft hier nicht als Handicap empfunden - im Gegensatz zu den Interviewten im bürgerlich-konservativen Lager. Diese nahmen seltener politische, sondern persönliche und kulturelle Differenzen als potenzielle Probleme wahr. Die Antworten der Interviews deuten zwar an, dass traditionelle Werte wie Religion und Familie für die bürgerlichen Politiker, egal ob Christen oder Muslime, stark ausgeprägt sind. Abseits der gemeinsamen konservativen politischen Einstellung kommt es jedoch zu Akzeptanzproblemen aufgrund der Religion und der Zugehörigkeit zu einem anderen Kulturkreis. Gerade in der CDU könnte dies zu Spannungen zwischen den Mitgliedern ohne und mit Migrationshintergrund, insbesondere Türkischstämmigen führen.

37 Vgl. Andreas M. Wüst / Dominic Heinz, a.a.O. (Fn. 3), S. 210. 